DD-LPS. The additional non-rearranged copies of EWSRI and DDIT3 could also be expected in a tumour with complex karyotype and are of not diagnostic value. The identification of a melanoma-compatible genotype or the demonstration of a shared mutation between metastatic and primary melanoma is a more robust criterion for the diagnosis of undifferentiated and dedifferentiated melanoma ${ }^{12}$ and we were able to demonstrate the co-occurrence of MDM2 gene amplification and $B R A F$ V600E mutation in the same tissue block. Interestingly, if the initial tissue core biopsy had targeted the myxoid DD-LPS-like areas, it is likely that this patient could have been initially misdiagnosed and potentially mismanaged. Increasing awareness of potentially conflicting molecular findings can help pathologists avoid pitfalls in interpretation. In particular, there is increasing demand to perform comprehensive diagnostic work-up and molecular testing on smaller biopsy material, and sampling factors in heterogeneous tumours may thus become more significant in terms of diagnosis and management.

Conflicts of interest and sources of funding: The authors state that there are no conflicts of interest to disclose.

Samer Yousef ${ }^{1}$, Christopher Joy $^{2}$, Shanta Velaiutham ${ }^{3}$, Fiona M. Maclean ${ }^{1,4,5}$, James Harraway ${ }^{2}$, Anthony J. Gill ${ }^{5,6,7}$, Ana Cristina Vargas ${ }^{1,5,7}$

${ }^{1}$ Department of Anatomical Pathology, Douglass Hanly Moir Pathology, NSW, Australia; ${ }^{2}$ Department of Cytogenetics, Sullivan Nicolades Pathology, Brisbane, Qld, Australia; ${ }^{3}$ Lake Macquarie Private Hospital, Gateshead, NSW, Australia; ${ }^{4}$ Department of Clinical Medicine, Faculty of Medicine, Health and Human Sciences, Macquarie University, NSW, Australia; ${ }^{5}$ Cancer Diagnosis and Pathology Group, Kolling Institute of Medical Research, Royal North Shore Hospital, NSW, Australia; ${ }^{6}$ NSW Health Pathology, Department of Anatomical Pathology, Royal North Shore Hospital, St Leonards, NSW, Australia; ${ }^{7}$ Faculty of Medicine, University of Sydney, NSW, Australia

Contact Dr Samer Yousef.

E-mail: sameryousefsamer@gmail.com

1. WHO Classification of Tumours Editorial Board. WHO Classification of Tumours: Soft Tissue and Bone Tumours. 5th ed, vol. 3. Lyon: IARC, 2020.

2. Thway K. Well-differentiated liposarcoma and dedifferentiated liposarcoma: an updated review. Semin Diagn Pathol 2019; 36: 112-21.

3. He X, Pang Z, Zhang X, et al. Consistent amplification of FRS2 and MDM2 in low-grade osteosarcoma: a genetic study of 22 cases with clinicopathologic analysis. Am J Surg Pathol 2018; 42: 1143-55.

4. Bode-Lesniewska B, Zhao J, Speel EJ, et al. Gains of 12q13-14 and overexpression of $\mathrm{mdm} 2$ are frequent findings in intimal sarcomas of the pulmonary artery. Virchows Arch 2001; 438: 57-65.

5. Hayward NK, Wilmott JS, Waddell N, et al. Whole-genome landscapes of major melanoma subtypes. Nature 2017; 545: 175-80.

6. Cancer Genome Atlas Network. Genomic classification of cutaneous melanoma. Cell 2015; 161: 1681-96.

7. Forschner A, Hilke F-J, Irina Bonzheim I, et al. MDM2, MDM4 and EGFR amplifications and hyperprogression in metastatic acral and mucosal melanoma. Cancers (Basel) 2020; 12: 540

8. Gradecki SE, Slingluff CL, Gru AA, et al. PRAME expression in 155 cases of metastatic melanoma. J Cutan Pathol 2021; 48: 479-85.

9. Albertsmeier M, Altendorf-Hofmann A, Lindner LH, et al. Cancer testis antigens and immunotherapy: expression of PRAME is associated with prognosis in soft tissue sarcoma. Cancers 2020; 12: 3612 .
10. Hemminger JA, Toland AE, Scharschmidt TJ, et al. Expression of cancer-testis antigens MAGEA1, MAGEA3, ACRBP, PRAME, SSX2, and CTAG2 in myxoid and round cell liposarcoma. Mod Pathol 2014; 27: $1238-45$.

11. Lura K, Kohashi K, Hotokebuchi Y, et al. Cancer-testis antigens PRAME and NY-ESO-1 correlate with tumour grade and poor prognosis in myxoid liposarcoma. J Pathol Clin Res 2015; 1: 144-59.

12. Agaimy A, Stoehr R, Hornung A, et al. Dedifferentiated and undifferentiated melanomas: report of 35 new cases with literature review and proposal of diagnostic criteria. Am J Surg Pathol 2021; 45: 240-54.

DOI: https://doi.org/10.1016/j.pathol.2021.05.096

\section{Agminated Spitz naevus with an activating HRAS Q61R mutation}

Sir,

Spitz naevi (SN) are benign melanocytic tumours arising preferentially after childhood. ${ }^{1}$ Under microscopic examination, $\mathrm{SN}$ appear as a well demarcated compound proliferation arranged in nests of epithelioid and spindled, often mildly pigmented melanocytes. Frequent epidermal hyperplasia is associated. Related oncogenes include mutually exclusive translocations involving $A L K, R O S 1, R E T, M E T$, $M E R T K, B R A F, L C K, N T R K, M A P 3 K 3$ and MAP3K8 genes. $^{2-6}$ In addition, HRAS exon 3 mutation is detected in a subset of SN harbouring desmoplastic morphology.

Although most SN appear as solitary tumours, rare cases of multiple SN either disseminated or clustered have been reported under the terminology agminated SN (ASN). ${ }^{8}$ Few genetic studies have been performed in this setting. Herein, we report a case of ASN with a previously undescribed Q61R HRAS mutation.

The study was conducted according to the Declaration of Helsinki and was approved by the research ethics committee of the Center Léon Bérard, Lyon, France (Ref: L20-72).

A 2-year-old boy presented with multiple pigmented papules restricted to the right side of the face (Fig. 1). The tumours arose after birth, increased rapidly in number (more than 40 at time of diagnosis) and size (ranging from 0.3 to 3.0 $\mathrm{cm}$ diameter). Physical examination of the skin revealed no additional skin, neurological or skeletal disorders. Surgical excision of the largest tumour was performed.

Microscopic examination revealed a wedged-shaped melanocytic proliferation (Fig. 2A). A few packed junctional nests of bland or spindled melanocytes were present associated with epidermal hyperplasia (Fig. 2B). No pagetoid spreading or ulceration were observed. In the underlying dermis, fascicles of medium-sized spindled melanocytes entrapped in a desmoplastic stroma were arranged in a plexiform growth pattern. Neither cytological atypia nor mitotic activity were seen (Fig. 2C).

Immunohistochemical study confirmed diffuse expression of the melanocytic marker MelanA, while no significant positivity was observed with antibodies against ALK, ROS1 and NTRK $1 / 2 / 3$ proteins.

Whole exome RNA-sequencing identified a HRAS Q61R mutation. No anomaly was present in other Spitz-related genes including ALK, ROS1, NTRK1, NTRK3, RET, MET, MERTK, $L C K, B R A F, M A P 3 K 3$, and $M A P 3 K 8$. Immunohistochemistry 

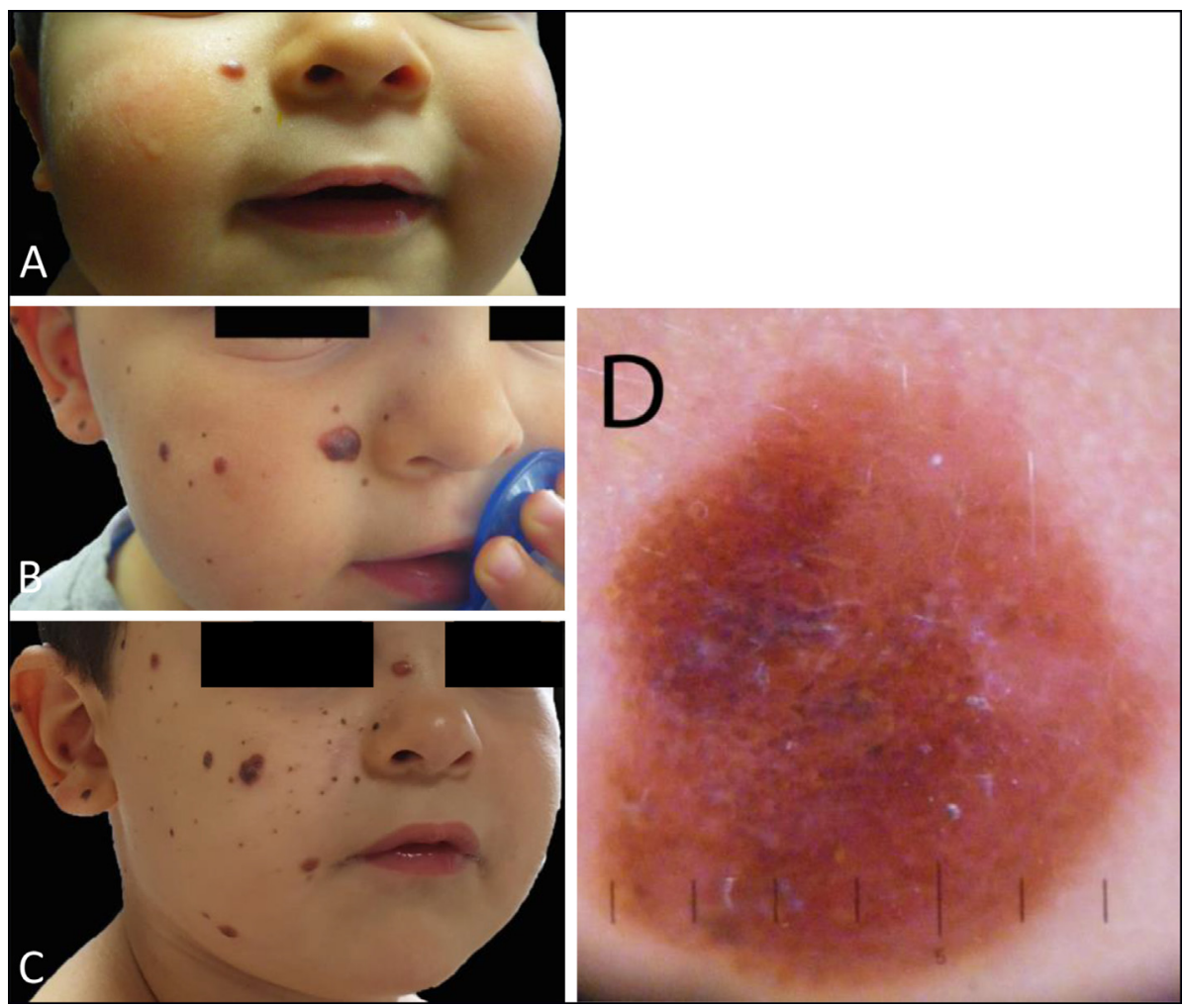

Fig. 1 (A-C) Clinical and (D) dermoscopic features of the case. (A) Clinical features at the age of 14, (B) 23 and (C) 33 months: the tumours arose after birth, increased rapidly in number (more than 40 at time of diagnosis) and size (ranging from 0.3 to $3.0 \mathrm{~cm}$ diameter). (D) Dermoscopic examination of the largest tumour revealed an inhomogeneous tumour harbouring two highly pigmentated areas and devoid of clearly distinguishable pattern (Fig. 1).

confirmed diffuse cytoplasmic positivity in tumoural melanocytes with the anti- $(N, H, K) R A S$ Q61R antibody (clone SP174, dilution 1/50; Abcam, UK) (Fig. 2D).

We report a case of ASN harbouring a previously undescribed Q61R HRAS mutation. Pathogenic RAS mutations promote cell growth through the permanent activation of the MAP-kinase pathway and therefore constitute an oncogenic driver in many cell lineages including melanocytes. HRAS mutations involving both exons 2 and 3 are commonly found in isolated SN. ${ }^{7}$ However, in ASN and especially those arising in the setting of naevus spilus, exon 2 G13R HRAS mutations have so far been described. ${ }^{9,10}$ Sarin et al. demonstrated that a post-zygotic G13R mutation of HRAS in a melanocytic progenitor resulted in naevus spilus formation. ${ }^{11}$ Furthermore, their study demonstrated that the proliferating Spitz melanocytes arising within the naevus spilus also presented an amplification of the mutated $H R A S$ gene, suggesting this supplementary anomaly was needed for SN tumour development. ${ }^{9}$ Although RNAseq did not allow us to assess the presence of a $H R A S$ amplification, the level of $H R A S$ expression observed in our case was extremely high compared to other melanocytic tumours present in our cluster (rank 2/568), with the 20 first ranked cases all being HRAS mutants (data not shown). This suggests that an HRAS amplification could be present in this tumour.

The papular naevus spilus syndrome is categorised among RASopathies, a heterogeneous group of diseases characterised by mosaicism of $R A S$ mutations occurring in various somatic cells (either keratinocytes, melanocytes or both) during embryonic development, ${ }^{11}$ resulting in various other conditions such as Schimmelpenning syndrome, epidermal and sebaceous naevi, and phakomatosis pigmentokeratotica.

Interestingly, since exon 3 HRAS mutations combined with $H R A S$ amplification are also detected in a subset of isolated $\mathrm{SN},{ }^{7}$ it is conceivable that similar post-zygotic Q61R HRAS mutations occurring in the melanocytic lineage during the embryonic life, represent a preliminary step to isolated $H R A S$ mutated SN development.

ASN have also been associated with other genetic anomalies. Goto et al. detected a complex rearrangement of TRPM1-PUM1-LCK in an ASN case arising in a giant congenital hyperpigmentated macule. ${ }^{5}$ More recently the same authors identified a GOPC-ROS1 mosaicism in two ASN cases. ${ }^{12}$ Clinically, a patchy pigmented macule was present in two of these three described cases. Both these anomalies involve a $5^{\prime}$-fused intact oncogenic tyrosine kinase domain. Fluorescence in situ hybridisation techniques confirmed the anomaly was also present in normalappearing melanocytes in the basal layers of the epidermis surrounding the Spitz proliferations. This association mimics the features of ASN arising in naevus spilus, suggesting supplementary anomalies are needed for further tumoural development. 

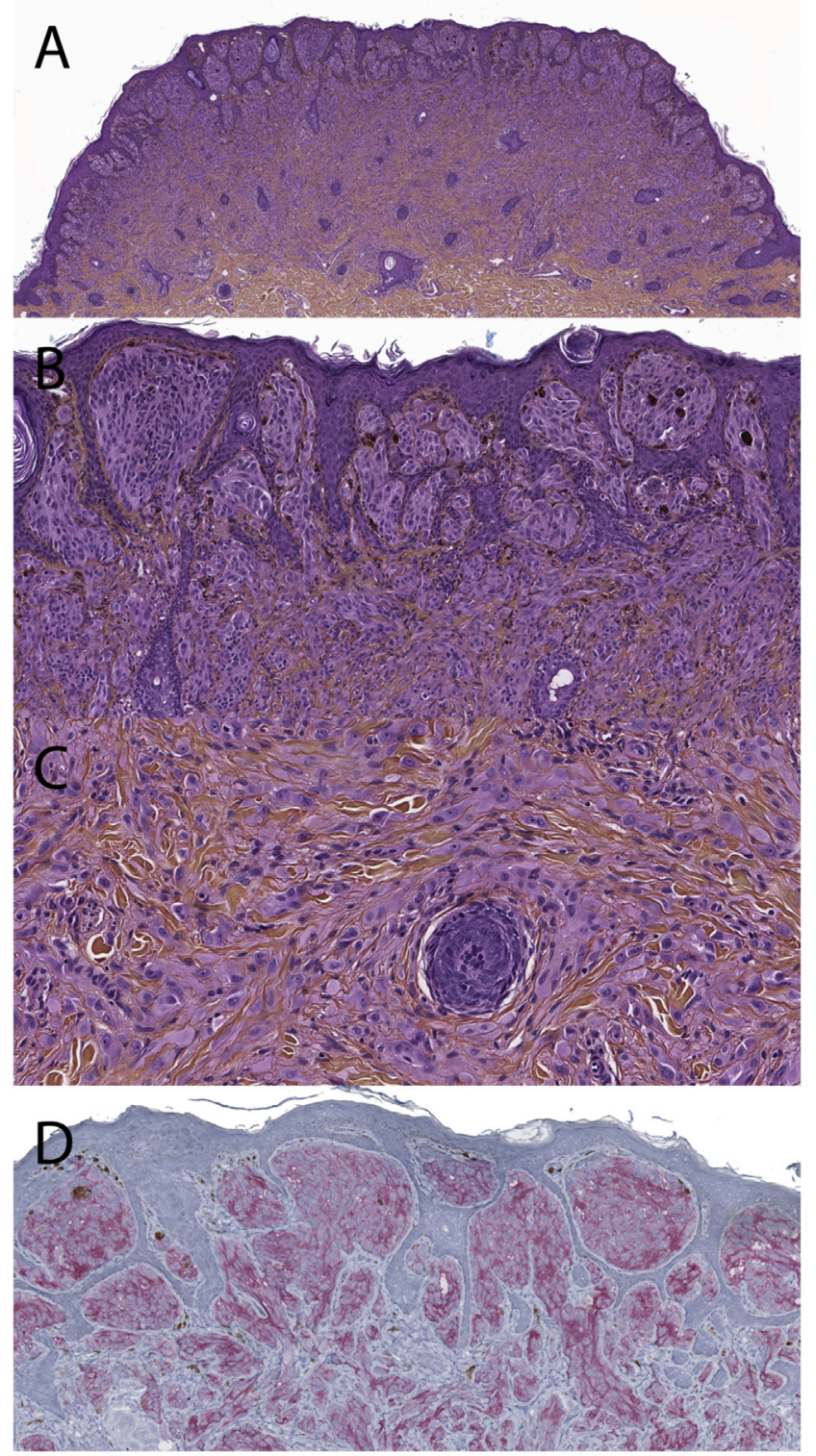

Fig. 2 (A-C) Microscopic and (D) immunohistochemical features of the Spitz naevus. (A-C) Microscopic examination revealed a dermal plexiform proliferation of spindle non-atypical melanocytes entrapped in a desmoplastic stroma (D) Immunohistochemistry demonstrated the RAS Q61R mutant in tumour cells

To conclude, our observation reports that in addition to G13R HRAS mutations, ROS1 and $L C K$ fusions, a postzygotic activating Q61R HRAS mutation in a melanocytic progenitor might constitute a preliminary step to ASN development.

Acknowledgement: The parents of the patient gave written informed consent for publication of this case and clinical photographs.

Conflicts of interest and sources of funding: The authors state that they have no significant relationships with, or financial interest in, any commercial companies pertaining to this study and there are no conflicts of interest to disclose.

Thibault Kervarrec $^{1,2, *}$, Clémence Briand ${ }^{3, *}$, Daniel Pissaloux ${ }^{1,4}$, Franck Tirode ${ }^{4}$, Claire AbasqThomas $^{3}$, Sylvie Fraitag ${ }^{5}$, Arnaud de la Fouchardière ${ }^{1,4}$
${ }^{1}$ Department of Pathology, Centre Hospitalier Universitaire de Tours, Tours, France; ${ }^{2}$ Department of Biopathology, Center Léon Bérard, Lyon, France; ${ }^{3}$ Department of Dermatology, Centre Hospitalier Universitaire de Brest, Brest, France; ${ }^{4}$ Université de Lyon, Université Claude Bernard Lyon 1, INSERM 1052, CNRS 5286, Centre Léon Bérard, Cancer Research Center of Lyon, Equipe Labellisée Ligue contre le Cancer, Lyon, France; ${ }^{5}$ Department of Pathology, Institut Imagine, APHP, Hôpital Universitaire Necker-Enfants Malades, Université Paris Descartes, Sorbonne Paris Cité, Paris, France; "These authors contributed equally

Contact: Dr Thibault Kervarrec.

E-mail: thibaultkervarrec@yahoo.fr

1. Elder DE, Massi D, Scolyer RA, Willemze R, editors. WHO Classification of Skin Tumours. 4th ed. Lyon: IARC, 2018.

2. Wiesner T, He J, Yelensky R, et al. Kinase fusions are frequent in Spitz tumours and spitzoid melanomas. Nat Commun 2014; 5: 3116.

3. Yeh I, Busam KJ, McCalmont TH, et al. Filigree-like rete ridges, lobulated nests, rosette-like structures, and exaggerated maturation characterize Spitz tumors with NTRK1 fusion. Am J Surg Pathol 2019; 43: 737-46.

4. Houlier A, Pissaloux D, Masse I, et al. Melanocytic tumors with MAP3K8 fusions: report of 33 cases with morphological-genetic correlations. Mod Pathol 2020; 33: 846-57.

5. Goto K, Pissaloux D, Durand L, Tirode F, Guillot B, de la Fouchardière A. Novel three-way complex rearrangement of TRPM1 PUM1-LCK in a case of agminated Spitz nevi arising in a giant congenital hyperpigmented macule. Pigment Cell Melanoma Res 2020 33: $767-72$

6. Yeh I, de la Fouchardiere A, Pissaloux D, et al. Clinical, histopathologic, and genomic features of Spitz tumors with ALK fusions. Am J Surg Pathol 2015: 39: 581-91.

7. Bastian BC, LeBoit PE, Pinkel D. Mutations and copy number increase of HRAS in Spitz nevi with distinctive histopathological features. Am J Pathol 2000; 157: 967-72.

8. Zayour M, Bolognia JL, Lazova R. Multiple Spitz nevi: a clinicopathologic study of 9 patients. J Am Acad Dermatol 2012; 67: 451-8 e2.

9. Sarin KY, Sun BK, Bangs CD, et al. Activating HRAS mutation in agminated Spitz nevi arising in a nevus spilus. JAMA Dermatol 2013 149: $1077-81$.

10. Pontoizeau J, Stefan A, Comoz F, et al. Agminated Spitz nevus arising in normal skin with redundant HRAS mutation. Eur J Dermatol 2017; 27: $73-4$.

11. Sarin KY, McNiff JM, Kwok S, Kim J, Khavari PA. Activating HRAS mutation in nevus spilus. J Invest Dermatol 2014; 134 1766-8.

12. Goto K, Pissaloux D, Kauer F, Huriet V, Tirode F, de la Fouchardière A. GOPC-ROS1 mosaicism in agminated Spitz nevi: report of two cases. Virchows Arch 2021; Mar 17: https://doi.org/10.1007/s00428-02002992-5.

DOI: https://doi.org/10.1016/j.pathol.2021.04.013

\section{SMARCA4-deficient anaplastic carcinoma arising in a primary retroperitoneal mucinous adenocarcinoma}

Sir,

Primary mucinous tumours arising in the retroperitoneum are uncommon and approximately half are histologically malignant, the remainder showing benign or borderline appearances according to ovarian-type criteria. ${ }^{1}$ As with primary ovarian neoplasms, retroperitoneal mucinous 